\title{
O que são campos rupestres e campos de altitude nos topos de montanha do Leste do Brasil?
}

\author{
MARCELO FERREIRA DE VASCONCELOS ${ }^{1}$
}

(recebido: 04 de Maio de 2009; aceito: 07 de abril de 2011)

\begin{abstract}
What are the campos rupestres and the campos de altitude in the eastern Brazilian mountaintops?). The campos rupestres and the campos de altitude from eastern Brazil occur in the mountaintops of the Espinhaço Range, Serra da Mantiqueira and Serra do Mar. Since there is some confusion on the characterization and about geographical limits of both vegetation types, the aim of this paper is to present a review of opinions about on those open environments, with discussions on the history of their nomenclature, physiognomic aspects and biogeographical affinities. Although the campos rupestres and the campos de altitude present some physiognomic and floristic similarities, both should be considered distinct vegetation types.
\end{abstract}

Key words - mountain biodiversity, phytogeography, phytophysiognomy

RESUMO - (O que são campos rupestres e campos de altitude nos topos de montanha do Leste do Brasil?). Os campos rupestres e os campos de altitude do leste do Brasil ocorrem nas partes mais elevadas da Cadeia do Espinhaço, da Serra da Mantiqueira e da Serra do Mar. Uma vez que há certa confusão sobre a caracterização e os limites geográficos destes dois tipos de vegetação, o objetivo deste trabalho é apresentar uma síntese das opiniões sobre tais ambientes campestres, discutindo sobre a história de suas denominações, localização geográfica, aspectos fisionômicos e afinidades biogeográficas. Embora os campos rupestres e os campos de altitude apresentem certas semelhanças fisionômicas e compartilhem alguns táxons vegetais, ambos os tipos de vegetação devem ser considerados distintos.

Palavras-chave - biodiversidade em montanhas, fitofisionomia, fitogeografia

\section{Introdução}

Nas últimas décadas, a flora das montanhas do Leste do Brasil tem atraído a atenção de diversos botânicos e muitos estudos sobre taxonomia vegetal e fitogeografia vêm sendo realizados nessas áreas altas, cobertas pelas vegetações dos campos rupestres e campos de altitude (Safford 1999a, Rapini et al. 2008). Entretanto, até o momento, há certa confusão sobre a caracterização e os limites geográficos desses dois tipos de vegetação. Desse modo, o objetivo deste trabalho é apresentar uma revisão sobre tais ambientes campestres, discutindo sobre a história de suas denominações, localização geográfica, aspectos fisionômicos e afinidades biogeográficas.

Os campos rupestres e de altitude do Leste do Brasil ocorrem nas partes mais elevadas dos três principais sistemas orográficos desta região: Cadeia do Espinhaço, Serra da Mantiqueira e Serra do Mar, sendo reconhecidos como importantes centros de endemismo da flora

1. Museu de Ciências Naturais, Pontifícia Universidade Católica de Minas Gerais, Avenida Dom José Gaspar, 290, Bairro Coração Eucarístico, Campus PUC Minas, 30535-901 Belo Horizonte, MG, Brasil.mfvasconcelos@gmail.com neotropical (Giulietti \& Pirani 1988, Eiten 1992, Giulietti et al. 1997, Safford 1999a, Rapini et al. 2008, Fiaschi \& Pirani 2009).

Em 1867, o botânico dinamarquês Johannes Eugenius Büllow Warming apresentou um mapa das regiões fitogeográficas do Brasil, no qual destacou, pela primeira vez, as vegetações de campos rupestres e de altitude como uma formação à parte do Cerrado e da Mata Atlântica, denominando esses tipos vegetacionais de "höjeste med en alpinsk flora bekloedte bjergtopper" ou "topos de montanha mais elevados cobertos por uma flora alpina" (Gomes et al. 2006).

O naturalista mineiro Alvaro Astolpho da Silveira, um dos pioneiros nos estudos taxonômicos da família Eriocaulaceae nas serras brasileiras, não aplicou uma denominação específica para as formações abertas desta região, usando termos como "campo", "campo limpo", "campo alpestre" e "campo alpino" (Silveira 1922, 1924). Entretanto, esse autor sugeriu nomes a serem aplicados a certos tipos de ambientes restritos a essas áreas, tais como "chusqueal", em referência a aglomerados de taquaras do gênero Chusquea nas partes mais altas da Serra do Caparaó (Silveira 1922), e "Campos de Eriocaulaceas", na Serra do Cipó (Silveira 1924). 
Sampaio (1938) e Mello-Barreto (1949) denominaram a vegetação aberta dos topos de montanha do Leste brasileiro de "campos alpinos", possivelmente seguindo a sugestão do mapa produzido anteriormente por Gonzaga de Campos (1912). Azevedo (1962) considerou esses campos, na região Sul do Estado de Minas Gerais, como uma única unidade, sugerindo o nome de "savana especial dos altos divisores".

Magalhães (1966) e Joly (1970) foram os primeiros a usar o termo "campos rupestres" para a vegetação ocorrente nos topos de montanha ao longo da Cadeia do Espinhaço. Entretanto, Joly (1970) considerou como "campos rupestres" tanto o tipo de vegetação ocorrente nas partes mais elevadas das serras de Minas Gerais e Goiás (sobre quartzito ou arenito), quanto nos topos das serras do Caparaó, dos Órgãos e do Itatiaia (sobre rochas ígneas ou metamórficas), sugerindo que não haveria diferença na classificação das vegetações abertas dos topos de montanha do Leste e do Centro do Brasil.

Rizzini (1979) dividiu a vegetação aberta dos topos de montanha do Leste brasileiro, objetos da presente revisão, em "campo de canga couraçada", para a vegetação que ocorre nos afloramentos ferruginosos do Quadrilátero Ferrífero de Minas Gerais; “campos quartzíticos", para campos sobre quartzito ao longo da Cadeia do Espinhaço e "campos altimontanos", para vegetações sobre rochas ígneas ou metamórficas ocorrentes nas Serras da Mantiqueira e do Mar.

Posteriormente, Ferri (1980) denominou os campos quartzíticos de "campos rupestres" e os campos altimontanos de "campos de altitude". Veloso et al. (1991) consideraram os campos rupestres e os campos de altitude como "refúgios vegetacionais" ou "relíquias de vegetação" por se tratarem de vegetações isoladas em um contexto completamente distinto da flora dominante nas regiões onde estes campos se localizam. Este tipo de classificação, entretanto, agrupou os dois tipos de vegetação sem levar em consideração suas diferenças de localização em sistemas orográficos, clima, substrato e, principalmente, afinidades biogeográficas. Outras denominações mais recentes, embora de uso restrito, são "complexos rupestres de altitude sobre rocha quartzítica", para os campos rupestres da Cadeia do Espinhaço, Chapadas dos Veadeiros e dos Guimarães, Roraima e em serras do Centro-Sul de Minas Gerais (e.g. Ibitipoca e Canastra), e "complexos rupestres de altitude sobre rocha ígnea", para os demais campos de altitude das Serras da Mantiqueira e do Mar (Benites et al. 2003, 2007).

Entretanto, as denominações de Ferri (1980) de "campos rupestres" e "campos de altitude" são as mais comumente citadas pelos botânicos e fitogeógrafos
(Giulietti \& Pirani 1988, Harley 1995, Giulietti et al. 1997, Safford 1999a, 2007, Fiaschi \& Pirani 2009). Seguindo as definições de Eiten (1992), os campos que ocorrem sobre afloramentos de óxido de ferro ("campo de canga couraçada", segundo Rizzini 1979), também são considerados como campos rupestres, por apresentarem semelhanças florísticas com os campos rupestres que ocorrem sobre quartzito ao longo da Cadeia do Espinhaço (Jacobi et al. 2007, Viana \& Lombardi 2007).

\section{Diferenciação dos campos rupestres e de altitude}

Em geral, os campos rupestres ocorrem principalmente acima de $900 \mathrm{~m}$ de altitude, em montanhas cujas rochas são de origem pré-cambriana que foram remodeladas por movimentos tectônicos a partir do Paleógeno, estando associados, principalmente, a afloramentos de quartzito, arenito e minério de ferro (King 1956, Joly 1970, Giulietti \& Pirani 1988, Eiten 1992, Alves \& Kolbelk 1994, Giulietti et al. 1997, Caiafa \& Silva 2005, Alves et al. 2007). Esses campos encontram-se distribuídos principalmente ao longo da Cadeia do Espinhaço, embora áreas isoladas desse tipo de vegetação também sejam encontradas nas serras do Brasil Central (e.g. Chapada dos Veadeiros e Serra dos Pirineus, ambas em Goiás, e Serra da Canastra, no Sudoeste de Minas Gerais) ou em montanhas da região de São João Del Rei (Serra do Lenheiro), Tiradentes (Serra de São José) e Itutinga, em Minas Gerais, estas três últimas consideradas como pertencentes à Serra da Mantiqueira, mas com geologia e afinidades florísticas mais relacionadas aos campos rupestres da Cadeia do Espinhaço (Giulietti \& Pirani 1988, Eiten 1992, Alves \& Kolbelk 1994, 2009, 2010, Gavilanes et al. 1995, Harley 1995, Giulietti et al. 1997, Alves et al. 2007, Rapini et al. 2008).

No Brasil, outras áreas com vegetações já tratadas como "rupestres" ou "campos rupestres", mas fora da região enfocada pela presente revisão, são: a Serra dos Carajás, no Pará, onde a vegetação cresce sobre canga (Silva et al. 1996), e a Serra da Jibóia, no Leste da Bahia, onde os campos rupestres ocorrem sobre substrato gnáissico-granítico (Queiroz et al. 1996). Ambas as regiões apresentam alguns táxons típicos dos campos rupestres do Leste brasileiro, tais como espécies de Vellozia Vand.

Já os campos de altitude são típicos dos pontos mais elevados de montanhas que se soergueram principalmente durante o Terciário (Serras do Mar e da Mantiqueira), estando geralmente situados acima de 
$1.500 \mathrm{~m}$ de altitude e associados a rochas ígneas ou metamórficas, como granito, gnaisse e, no caso particular de Itatiaia, nefelino-sienito (Segadas-Vianna 1965, Petri \& Fúlfaro 1988, Martinelli \& Orleans e Bragança 1996, Giulietti et al. 1997, Safford 1999a, Caiafa \& Silva 2005, Alves et al. 2007). Uma exceção é representada pelos campos de altitude do Parque Estadual da Serra do Mar, no Núcleo Curucutu, que ocorrem em cotas mais baixas, entre $750 \mathrm{~m}$ e $850 \mathrm{~m}$ de altitude (Garcia \& Pirani 2003, 2005). Estes autores sugeriram que a altitude não seria determinante para a ocorrência desse tipo de vegetação, mas uma combinação de fatores, tais como condições topográficas, proximidade do oceano e circulação atmosférica.

Com relação aos domínios vegetacionais, em geral, os campos rupestres da Cadeia do Espinhaço estão situados em áreas de transição entre o Cerrado, a Caatinga e a Mata Atlântica, enquanto os campos de altitude das Serras do Mar e da Mantiqueira encontram-se totalmente inseridos na região da Mata Atlântica (Eiten 1992, Harley 1995, Martinelli \& Orleans e Bragança 1996, Giulietti et al. 1997, Safford 1999a, Caiafa \& Silva 2005).

A maioria das localidades de altitudes elevadas da Cadeia do Espinhaço é representada por campos rupestres e todos os topos da Serra do Mar são cobertas por campos de altitude. Na Serra da Mantiqueira, conforme acima mencionado, as Serras do Lenheiro e de São José, localizadas no centro-sul de Minas Gerais, são formadas por quartzito e consideradas como localidades de campos rupestres. Porém, duas localidades, as Serras do Caraça e do Ibitipoca, ambas em Minas Gerais, aparentam ser caracterizadas pelos dois tipos de vegetação.

$\mathrm{Na}$ Serra do Caraça, no extremo meridional da Cadeia do Espinhaço, os picos mais elevados (do Sol e do Inficionado), que ultrapassam $2.000 \mathrm{~m}$ de altitude, possuem flora típica de campos rupestres sobre afloramentos rochosos, embora vários táxons vegetais típicos dos campos de altitude ocorram em áreas onde há algum tipo de solo ou em terrenos brejosos (Vasconcelos 2000).

Apesar da maioria das localidades da Serra da Mantiqueira ser representada por formações cristalinas, o Ibitipoca é uma serra quartzítica (Dias et al. 2002, Benites et al. 2007). De fato, não há consenso quanto à classificação da vegetação campestre dessa região. Giulietti \& Pirani (1988), Rapini et al. (2008) e Alves \& Kolbek (2010) consideraram os campos localizados na Serra do Ibitipoca como rupestres. Andrade \& Sousa (1995) dividiram a vegetação campestre do Ibitipoca em três formações: campo graminoso, campos rupestres e campo com arbustos e arvoretas. Pires (1997) também sugeriu a existência de três tipos de vegetação campestre na região: campos rupestres, campos rupestres arborizados e campo gramíneo-lenhoso. Rodela (1998) reconheceu quatro tipos de vegetação aberta no Ibitipoca: cerrado de altitude, campo rupestre, campo herbáceograminoso e campo encharcável. Dias et al. (2002) consideraram que os campos de Ibitipoca são formados por um mosaico, sendo os campos rupestres típicos de áreas de afloramentos rochosos ou de solos rasos e os campos de altitude ocorrentes em locais onde o solo é mais profundo.

Uma classificação semelhante àquela de Dias et al. (2002) foi apresentada por Oliveira Filho \& Fluminhan Filho (1999) para a Serra do Carrapato, em Lavras (Minas Gerais), de modo que áreas de vegetação herbácea associada a afloramentos quartzíticos foram tratadas como sendo campos rupestres, enquanto áreas sem afloramentos foram consideradas como campos de altitude. Entretanto, outros autores que realizaram estudos florísticos nessa mesma área, identificaram apenas a vegetação de campos rupestres nas partes mais elevadas dessa serra (Gavilanes \& Brandão 1991), o que condiz com a vegetação já identificada para as serras adjacentes àquela região (Itutinga, São João Del Rei e Tiradentes).

Assim, essas classificações não são as mesmas usadas correntemente pelos botânicos e fitogeógrafos, conforme acima mencionado, principalmente porque o que Oliveira Filho \& Fluminhan Filho (1999) consideraram como "campo de altitude" é um tipo de vegetação bastante distinta da ocorrente nas altas montanhas de rochas ígneas ou metamórficas das Serras da Mantiqueira e do Mar (Safford 1999a, Ribeiro et al. 2007). Ao contrário dessas últimas, a flora desses "campos de altitude" apresenta ocorrência marcante de espécies do Cerrado (ver exemplos em Oliveira Filho \& Fluminhan Filho 1999).

Da mesma forma, a denominação de "campos de altitude" para a vegetação campestre ocorrente nas partes mais elevadas do Maciço do Urucum, em Corumbá, Mato Grosso do Sul (conforme Tomas et al. 2010), também deve ser evitada, pois suas afinidades florísticas estão relacionadas ao Cerrado (Lehn et al. 2008) e não aos típicos campos de altitude das Serras do Mar e da Mantiqueira. Uma vez que já existe denominação apropriada para a vegetação ocorrente no alto do Maciço do Urucum - "campo limpo", conforme Ribeiro \& Walter (1998) - a aplicação errônea de nomes já cunhados para outros tipos de vegetação ("campos de altitude") serve apenas para causar mais confusão ao leitor desavisado. 
Tanto os campos rupestres da Cadeia do Espinhaço quanto os campos de altitude das Serras do Mar e da Mantiqueira são representados por um conjunto de comunidades predominantemente herbáceo-arbustivas que variam em função do relevo, microclima, profundidade do solo e natureza do substrato, o que dá um caráter de mosaico a estes tipos de vegetação (Segadas-Vianna 1965, Giulietti \& Pirani 1988, Giulietti et al. 1997, Safford 1999a, Conceição \& Pirani 2005, Alves et al. 2007, Rapini et al. 2008). Assim, ambos os tipos de vegetação apresentam uma ampla variedade de fisionomias, desde áreas abertas cobertas por gramíneas e outras ervas, a habitats com adensamento de arbustos e pequenas árvores, com ou sem a presença de afloramentos rochosos.

Apesar de os campos rupestres e os de altitude apresentarem paisagens, até certo ponto, semelhantes, além de compartilharem gêneros e espécies de plantas, os dois tipos de vegetação mostram diferenças com relação às afinidades biogeográficas de suas floras (Rizzini 1979, Giulietti \& Pirani 1988, Eiten 1992, Pirani et al. 1994, Harley 1995, Martinelli \& Orleans e Bragança 1996, Giulietti et al. 1997, Safford 1999a, 2007, Garcia \& Pirani 2005, Alves et al. 2007, Fiaschi \& Pirani 2009). Neste aspecto, a flora dos campos rupestres da Cadeia do Espinhaço apresenta afinidades com as das serras do Brasil Central, do Escudo das Guianas (Tepuis) e das restingas litorâneas ao longo do Oceano Atlântico (Giulietti \& Pirani 1988, Alves et al. 2007, Rapini et al. 2008, Fiaschi \& Pirani 2009). Além disso, poucos elementos andinos ou da região meridional do Brasil também são encontrados nos campos rupestres (Giulietti et al. 1997 versus Alves \& Kolbek 2010).

Por outro lado, a flora dos campos de altitude das Serras do Mar e da Mantiqueira mostra uma notável afinidade com aquelas da região andino-patagônica e das serras do sul do Brasil (Martinelli \& Orleans e Bragança 1996, Safford 1999a, 2007). Por este motivo, os campos de altitude foram considerados "páramos brasileiros", devido às suas semelhanças florísticas, fisionômicas, climáticas e edáficas com as regiões mais altas dos Andes e das montanhas da América Central (Safford 1999a, b, 2007). Apesar disto, Ribeiro et al. (2007) ponderaram que os campos de altitude apresentam maior efeito da sazonalidade e alta riqueza de espécies, sendo muitas delas endêmicas restritas.

Alves \& Kolbek (2010), realizaram análises multivariadas baseadas em 25 levantamentos florísticos, demonstrando não ser possível diferenciar as vegetações de campos rupestres e de campos de altitude em nível de gênero, embora estimem que 1.200 espécies sejam endêmicas dos campos rupestres e cerca de 100, restritas aos campos de altitude, o que evidencia que ambos os tipos de vegetação sejam diferenciados com base na composição de espécies. Entretanto, ainda há necessidade de se ajustar alguns gêneros considerados por Alves \& Kolbek (2010) como endêmicos e/ou característicos de cada tipo de vegetação. Por exemplo, esses autores não incluíram nas análises o gênero Fernseea Baker (Bromeliaceae), endêmico dos campos de altitude e representado por duas espécies (Martinelli et al. 2009). Por outro lado, consideraram o gênero Prepusa Mart. (Gentianaceae) como endêmico dos campos de altitude, com três espécies, embora este número seja de quatro espécies restritas a este tipo de vegetação, além de omitirem a ocorrência de $P$. montana Mart., endêmica dos campos rupestres da Bahia (Calió et al. 2008). Além disso, dois gêneros endêmicos dos campos rupestres, da família Asteraceae, não foram considerados nas análises destes autores: Semiria D. J. N. Hind (Hind 1999) e Catolesia D. J. N. Hind (Hind 2000).

Assim, embora os campos rupestres e os campos de altitude apresentem certas semelhanças fisionômicas e compartilhem alguns táxons vegetais, esses tipos de vegetação devem ser considerados distintos, notadamente pela distinção dos substratos de natureza geológica, pelas floras com composição específica diferenciada e afinidades biogeográficas contrastantes, além de estarem situados em domínios fitogeográficos diferentes. As localidades que parecem abrigar ambos os tipos de vegetação, a exemplo dos altos picos da Serra do Caraça e da Serra do Ibitipoca, devem ser estudadas com mais detalhes, a fim de se refinar o conhecimento sobre suas afinidades biogeográficas.

Agradecimentos - Agradeço aos Drs. José Rubens Pirani, Alexandre Salino e a três revisores anônimos pela revisão crítica do manuscrito. O Dr. Jon Fjeldså traduziu informações do mapa em dinamarquês com as regiões fitogeográficas do Brasil, produzido por Warming no século XIX. Este estudo foi desenvolvido com o apoio da Coordenação de Aperfeiçoamento de Pessoal de Nível Superior (Capes) que me forneceu uma bolsa de doutorado no Programa de Pós-graduação em Ecologia, Conservação e Manejo de Vida Silvestre da Universidade Federal de Minas Gerais (UFMG).

\section{Referências bibliográficas}

ALVES, R.J.V. \& KOLBEK, J. 1994. Plant species endemism in savanna vegetation on table mountains (campo rupestre) in Brazil. Vegetatio 113:125-139. 
ALVES, R.J.V. \& KOLBEK, J. 2009. Summit vascular flora of Serra de São José, Minas Gerais, Brazil. Check List 5:35-73.

ALVES, R.J.V. \& KOLBEK, J. 2010. Can campo rupestre vegetation be floristically delimited based on vascular plant genera? Plant Ecology 207:67-79.

ALVES, R.J.V., CARDIN, L. \& KROPF, M.S. 2007. Angiosperm disjunction "campos rupestres - restingas": a re-evaluation. Acta Botanica Brasilica 21:675-685.

ANDRADE, P.M. \& SOUSA, H.C. 1995. Contribuição ao conhecimento da vegetação do Parque Estadual de Ibitipoca, Lima Duarte, Minas Gerais. Revista Árvore 19:249-261.

AZEVEDO, L.G. 1962. Tipos de vegetação do sul de Minas e campos da Mantiqueira (Brasil). Anais da Academia Brasileira de Ciências 34:225-234.

BENITES, V.M., CAIAFA, A.N., MENDONÇA, E.S., SCHAEFER, C.E. \& KER, J.C. 2003. Solos e vegetação nos complexos rupestres de altitude da Mantiqueira e do Espinhaço. Floresta e Ambiente 10:76-85.

BENITES, V.M., SCHAEFER, C.E.G.R., SIMAS, F.N.B. \& SANTOS, H.G. 2007. Soils associated with rock outcrops in the Brazilian mountain ranges Mantiqueira and Espinhaço. Revista Brasileira de Botânica 30: 569-577.

CAIAFA, A.N. \& SILVA, A.F. 2005. Composição florística e espectro biológico de um campo de altitude no Parque Estadual da Serra do Brigadeiro, Minas Gerais - Brasil. Rodriguésia 56:163-173.

CALIÓ, M.F., PIRANI, J.R. \& STRUWE, L. 2008. Morphology-based phylogeny and revision of Prepusa and Senaea (Gentianaceae: Helieae) - rare endemics from eastern Brazil. Kew Bulletin 63:169-191.

CONCEIÇÃO, A.A. \& PIRANI, J.R. 2005. Delimitação de habitats em campos rupestres na Chapada Diamantina, Bahia: substratos, composição florística e aspectos estruturais. Boletim de Botânica da Universidade de São Paulo 23:85-111.

DIAS, H.C.T., FERNANDES FILHO, E.I., SCHAEFER, C.E.G.R., FONTES, L.E.F. \& VENTORIM, L.B. 2002. Geoambientes do Parque Estadual do Ibitipoca, município de Lima Duarte-MG. Revista Árvore 26: 777-786.

EITEN, G. 1992. Natural Brazilian vegetation types and their causes. Anais da Academia Brasileira de Ciências 64:35-65.

FERRI, M.G. 1980. Vegetação brasileira. Editora da Universidade de São Paulo, São Paulo.

FIASCHI, P. \& PIRANI, J.R. 2009. Review of plant biogeographic studies in Brazil. Journal of Systematics and Evolution 47:477-496.

GARCIA, R.J.F. \& PIRANI, J.R. 2003. Revisão sobre o diagnóstico da vegetação campestre junto à crista de serras, no Parque Estadual da Serra do Mar, São Paulo, SP, Brasil. Hoehnea 30:217-241.
GARCIA, R.J.F. \& PIRANI, J.R. 2005. Análise florística, ecológica e fitogeográfica do Núcleo Curucutu, Parque Estadual da Serra do Mar (São Paulo, SP), com ênfase nos campos junto à crista da Serra do Mar. Hoehnea 32:1-48.

GAVILANES, M.L. \& BRANDÃO, M. 1991. Flórula da Reserva Biológica Municipal do Poço Bonito, Lavras, MG. II - formação campo rupestre. Daphne 2:7-18.

GAVILANES, M.L., BRANDÃO, M., LACA-BUENDIA, J.P. \& ARAUJO, M.G. 1995. Cobertura vegetal da Serra de São José, MG, municípios de São João Del Rei e Tiradentes. Daphne 5:40-72.

GIULIETTI, A.M. \& PIRANI, J.R. 1988. Patterns of geographic distribution of some plant species from the Espinhaço Range, Minas Gerais and Bahia, Brazil. In Proceedings of a workshop on Neotropical distribution patterns (P.E. Vanzolini \& W.R. Heyer, eds.). Academia Brasileira de Ciências, Rio de Janeiro, p.39-69.

GIULIETTI, A.M., PIRANI, J.R. \& HARLEY, R.M. 1997. Espinhaço Range region, eastern Brazil. In Centres of plant diversity: a guide and strategy for their conservation (S.D. Davis, V.H. Heywood, O. Herrera-MacBryde, J. Villa-Lobos \& A.C. Hamilton, eds.). Information Press, Oxford, v.3, p.397-404.

GOMES, M.C.A.,HOLTEN, B.\& STERLL, M.2006.Acanção das palmeiras: Eugenius Warming, um jovem botânico no Brasil. Fundação João Pinheiro, Belo Horizonte.

GONZAGA DE CAMPOS, L.F. 1912. Mappa Florestal. Ministerio da Agricultura, Industria e Commercio, Rio de Janeiro.

HARLEY, R.M. 1995. Introduction. In Flora of the Pico das Almas, Chapada Diamantina - Bahia, Brazil (B.L. Stannard, Y.B. Harvey \& R.M. Harley, eds.). Royal Botanic Gardens, Kew, p.1-42.

HIND, D.J.N. 1999. A new genus, Semiria (Compositae: Eupatorieae), and a discussion of its affinities within the subtribe Gyptidinae of Bahia, Brazil. Kew Bulletin 54:425-435.

HIND, D.J.N. 2000. A new genus, Catolesia (Compositae: Eupatorieae), and a discussion of its affinities within the subtribe Gyptidinae of Bahia, Brazil. Kew Bulletin 55:941-948.

JACOBI, C.M., CARMO, F.F., VINCENT, R.C. \& STEHMANN, J.R. 2007. Plant communities on ironstone outcrops: a diverse and endangered Brazilian ecosystem. Biodiversity and Conservation 16:2185-2200.

JOLY, A.B. 1970. Conheça a vegetação brasileira. Editora da Universidade de São Paulo e Polígono, São Paulo.

KING, L.C. 1956. A geomorfologia do Brasil oriental. Revista Brasileira de Geografia 18:147-265.

LEHN, C.R., ALVES, F.M. \& DAMASCENO-JUNIOR, G.A. 2008. Florística e fitossociologia de uma área de cerrado sensu stricto na região da borda oeste do Pantanal, Corumbá, MS, Brasil. Pesquisas, Botânica 59:129-142. 
MAGALHÃES, G.M. 1966. Sobre os cerrados de Minas Gerais. Anais da Academia Brasileira de Ciências 38: 59-70.

MARTINELLI, G. \& ORLEANS E BRAGANÇA, J. 1996. Campos de altitude. Editora Index, Rio de Janeiro.

MARTINELLI, G., VIEIRA, C.M., LEITMAN, P., COSTA, A.F. \& FORZZA, R.C. 2009. Bromeliaceae. In Plantas da Floresta Atlântica (J.R. Stehmann, R.C. Forzza, A. Salino, M. Sobral, D.P. Costa \& L.H.Y. Kamino, eds.). Jardim Botânico do Rio de Janeiro, Rio de Janeiro, p.186-204.

MELLO-BARRETO, H.L. 1949. Regiões fitogeográficas de Minas Gerais. Boletim de Geografia 14:14-28.

OLIVEIRA FILHO, A.T. \& FLUMINHAN FILHO, M. 1999. Ecologia da vegetação do Parque Florestal Quedas do Rio Bonito. Cerne 5:51-64.

PETRI, S. \& FÚLFARO, V.J. 1988. Geologia do Brasil (Fanerozóico). Editora da Universidade de São Paulo, São Paulo.

PIRANI, J.R., GIULIETTI, A.M., MELLO-SILVA, R. \& MEGURO, M. 1994. Checklist and patterns of geographic distribution of the vegetation of Serra do Ambrósio, Minas Gerais, Brazil. Revista Brasileira de Botânica 17:133-147.

PIRES, F.R.S. 1997. Aspectos fitofisionômicos e vegetacionais do Parque Estadual do Ibitipoca, Minas Gerais, Brasil. In Anais do I Seminário de Pesquisa sobre o Parque Estadual do Ibitipoca (C.G. Rocha, coord.). Núcleo de Pesquisa em Zoneamento Ambiental da Universidade Federal de Juiz de Fora, Juiz de Fora, p.51-60.

QUEIROZ, L.P., SENA, T.S.N. \& COSTA, M.J.S.L. 1996. Flora vascular da Serra da Jibóia, Santa Terezinha Bahia. I: o campo rupestre. Sitientibus 15:27-40.

RAPINI, A., RIBEIRO, P.L., LAMBERT, S. \& PIRANI, J.R. 2008. A flora dos campos rupestres da Cadeia do Espinhaço. Megadiversidade 4:16-24.

RIBEIRO, J.F. \& WALTER, B.M.T. 1998. Fitofisionomias do bioma Cerrado. In Cerrado: ambiente e flora (S.M. Sano \& S.P. Almeida, eds.). Embrapa - CPAC, Planaltina, p.89-166.

RIBEIRO, K.T., MEDINA, B.M.O. \& SCARANO, F.R. 2007. Species composition and biogeographic relations of the rock outcrop flora on the high plateau of Itatiaia, SE-Brazil. Revista Brasileira de Botânica 30:623-639.

RIZZINI, C.T. 1979. Tratado de fitogeografia do Brasil: aspectos sociológicos e florísticos. Editora Hucitec, São Paulo, v.2.
RODELA, L.C. 1998. Cerrados de altitude e campos rupestres do Parque Estadual de Ibitipoca, sudeste de Minas Gerais: distribuição e florística por subfisionomias da vegetação. Revista do Departamento de Geografia USP 12:163-189.

SAFFORD, H.D. 1999a. Brazilian páramos I. An introduction to the physical environment and vegetation of the campos de altitude. Journal of Biogeography 26:693-712.

SAFFORD, H.D. 1999b. Brazilian páramos II. Macro- and mesoclimate of the campos de altitude and affinities with high mountain climates of the tropical Andes and Costa Rica. Journal of Biogeography 26:713-737.

SAFFORD, H.D. 2007. Brazilian páramos IV. Phytogeography of the campos de altitude. Journal of Biogeography 34:1701-1722.

SAMPAIO, A.J. 1938. Phytogeografia do Brasil. Companhia Editora Nacional, Rio de Janeiro.

SEGADAS-VIANNA, F. 1965. Ecology of the Itatiaia Range, southeastern Brazil. I - altitudinal zonation of the vegetation. Arquivos do Museu Nacional 53:7-30.

SILVA, M.F.F., SECCO, R.S. \& LOBO, M.G.A. 1996. Aspectos ecológicos da vegetação rupestre da Serra dos Carajás, estado do Pará, Brasil. Acta Amazonica 26:17-44.

SILVEIRA, A.A. 1922. Memorias chorographicas. Imprensa Official, Belo Horizonte, v.1.

SILVEIRA, A.A. 1924. Narrativas e memorias. Imprensa Official, Belo Horizonte, v.2.

TOMAS, W.M., ISHII, I.H., STRUSSMANN, C., NUNES, A.P., SALIS, S.M., CAMPOS, Z., FERREIRA, V.L., BORDIGNON, M.O., BARROS, A.T.M. \& PADILHA, D.R.C. 2010. Borda oeste do Pantanal e Maciço do Urucum em Corumbá, MS: área prioritária para conservação da biodiversidade. In Anais do V Simpósio sobre Recursos Naturais e Socioeconômicos do Pantanal (W.M. Tomas, coord.). Embrapa Pantanal, Corumbá, p.1-6.

VASCONCELOS, M.F. 2000. Reserva do Caraça: história, vegetação e fauna. Aves 1:3-7.

VELOSO, H.P., RANGEL FILHO, A.L.R. \& LIMA, J.C.A. 1991. Classificação da vegetação brasileira, adaptada a um sistema universal. Instituto Brasileiro de Geografia e Estatística, Rio de Janeiro.

VIANA, P.L. \& LOMBARDI, J.A. 2007. Florística e caracterização dos campos rupestres sobre canga na Serra da Calçada, Minas Gerais, Brasil. Rodriguésia 58:159-177. 